\title{
cmaJOPEN
}

\section{Uptake of influenza vaccination among persons with inflammatory bowel disease, multiple sclerosis or rheumatoid arthritis: a population-based matched cohort study}

\author{
Ruth Ann Marrie MD PhD, Randy Walld BSc, James M. Bolton MD, Jitender Sareen MD, \\ Scott B. Patten MD PhD, Alexander Singer MD, Lisa M. Lix PhD, Carol A. Hitchon MD MSc, \\ James J. Marriott MD MSc, Renée El-Gabalawy PhD, Alan Katz MBChB MSc, John D. Fisk PhD, \\ Charles N. Bernstein MD; for the CIHR Team in Defining the Burden and Managing the Effects \\ of Psychiatric Comorbidity in Chronic Immunoinflammatory Disease
}

\section{Abstract}

Background: Individuals with immune-mediated inflammatory diseases, such as inflammatory bowel disease, multiple sclerosis and rheumatoid arthritis, are at increased risk for influenza and related complications. We examined and compared the uptake of influenza vaccination among people with and without these diseases, as well as the influence of psychiatric comorbidity on vaccine uptake.

Methods: Using administrative data from Apr. 1, 1984, to Mar. 31, 2016, we conducted a retrospective matched cohort study in Manitoba, Canada. We matched persons 18 years of age or older who had a diagnosis of inflammatory bowel disease, multiple sclerosis or rheumatoid arthritis (the immune-mediated inflammatory disease cohorts) with persons who did not have these diagnoses (the control cohorts) on age, sex and region. We then identified cohort members with any mood or anxiety disorder (depression, anxiety disorders, bipolar disorder). We identified influenza vaccinations through billing codes. Using binomial regression, we modelled the difference in the proportion of the immune-mediated inflammatory disease and matched cohorts vaccinated annually, with adjustment for sociodemographic characteristics, comorbidity and immune therapy. We tested additive interaction effects between a person's cohort and presence of a mood or anxiety disorder.

Results: We identified 32880 individuals with 1 or more immune-mediated inflammatory diseases (10 148 with inflammatory bowel disease, 6158 with multiple sclerosis and 16975 with rheumatoid arthritis) and a total of 164152 controls. In fiscal year 2015,8668 (41.3\%, 95\% confidence interval [Cl] $40.6 \%$ to $42.0 \%$ ) of the 20982 persons with an immune-mediated inflammatory disease received an influenza vaccination, a rate higher than among controls (35 238 of $104634 ; 33.7 \%, 95 \% \mathrm{Cl} 33.4 \%$ to $34.0 \%$ ). After adjustment, participants with an immune-mediated inflammatory disease but no mood or anxiety disorder had $6.44 \%(95 \% \mathrm{Cl}$ $5.79 \%$ to $7.10 \%$ ) greater uptake of vaccination than participants without such a disease. Among participants without an immunemediated inflammatory disease, having a mood or anxiety disorder was associated with $4.54 \%(95 \% \mathrm{Cl} 4.20 \%$ to $4.89 \%)$ greater uptake of vaccination. However, we observed a subadditive interaction between immune-mediated inflammatory disease and psychiatric status $(-1.38 \%, 95 \% \mathrm{Cl}-2.26 \%$ to $-0.50 \%)$.

Interpretation: Uptake of influenza vaccination was consistently low in populations with immune-mediated inflammatory disease, and although psychiatric morbidity is associated with greater vaccine uptake by Manitobans, it negatively interacts with these diseases to reduce uptake. Changes in care delivery are needed to mitigate this gap in care.

\footnotetext{
T ndividuals affected by immune-mediated inflammatory diseases (referred to hereafter as immune-mediated diseases), such as inflammatory bowel disease, multiple sclerosis and rheumatoid arthritis, share an increased risk for influenza and related complications; ${ }^{1}$ therefore, prevention of influenza is important. Effective implementation of influenza vaccination strategies requires knowledge of vaccine uptake or use among target populations. However, our understanding of influenza vaccine uptake in populations with immune-mediated diseases has been limited by studies with small sample sizes, selection bias, cross-sectional
}

designs, variable study durations and variable study periods. Prior findings regarding vaccine uptake among persons with immune-mediated diseases have been highly variable. ${ }^{2-5} \mathrm{~A}$

Competing interests: See the end of the article.

This article has been peer reviewed.

Correspondence to: Ruth Ann Marrie, rmarrie@ hsc.mb.ca

CMAJ Open 2021. DOI:10.9778/cmajo.20200105 
population-based assessment of influenza vaccine uptake by persons with immune-mediated diseases was needed to clarify this potential gap in preventive care.

Some psychiatric disorders, such as schizophrenia, are associated with lower uptake of preventive health care such as vaccinations. ${ }^{6,7}$ It is unknown whether mood and anxiety disorders have a similar effect on preventive health behaviours; however, it is known that they affect people with immunemediated diseases more often than people without such diseases. ${ }^{8}$ Small studies have suggested that the presence of any comorbidity may increase vaccination uptake, ${ }^{5}$ but others reported no association. ${ }^{9}$

We compared the uptake of influenza vaccination in population-based cohorts with inflammatory bowel disease, multiple sclerosis or rheumatoid arthritis and in matched cohorts without these diseases; we then evaluated the influence of mood and anxiety disorders on vaccine uptake. We hypothesized that uptake would be higher in the cohorts with immune diseases than among those without immune disease, and that psychiatric disorders would be associated with reduced uptake. We focused on immune-mediated diseases, with care provided by different specialists, that are highly prevalent in Canada, ${ }^{10-12}$ affect individuals across the age spectrum and affect different organ systems.

\section{Methods}

\section{Design and setting}

We conducted this retrospective matched cohort study in Manitoba, Canada, where publicly funded health care is provided for medically necessary services. Manitoba Health, the provincial health department, maintains health services databases, and we accessed data for the period Apr. 1, 1984, to Mar. 31, 2016.

\section{Data sources}

We accessed high-quality databases housed in the Manitoba Population Research Data Repository at the Manitoba Centre for Health Policy, including the population registry, the Discharge Abstract Database, the medical services database and the Drug Program Information Network database. ${ }^{13-15} \mathrm{We}$ linked these databases at the individual level using an encrypted unique identifier.

For provincial residents who are eligible to receive health services, the population registry captures sex, dates of birth and death, health care coverage and region of residence (by postal code). The Discharge Abstract Database captures hospital admissions, related dates and up to 25 diagnoses. Until Mar. 31, 2004, diagnoses were recorded using the International Classification of Diseases, 9th Revision (clinical modification) (ICD-9-CM) codes; ${ }^{16}$ thereafter, diagnoses were recorded by International Statistical Classification of Diseases and Related Health Problems, 10th Revision, enhanced Canadian version codes. ${ }^{17}$ The medical services database captures service date, 1 diagnosis assigned using ICD-9-CM codes and tariff (i.e., billing) codes (including codes for vaccinations). Since 1995, the Drug Program
Information Network database has captured outpatient prescription dispensations, including the drug identification number (which links to the World Health Organization's Anatomic Therapeutic Chemical Classification System ${ }^{18}$ ) and the date of dispensation.

\section{Study populations}

Using validated case definitions, we identified all Manitobans aged 18 years or older who had inflammatory bowel disease (including Crohn disease and ulcerative colitis), multiple sclerosis or rheumatoid arthritis (Appendix 1, Table e1, available at www.cmajopen.ca/content/9/2/E510/suppl/ DC1). ${ }^{19-21}$ For each case, we defined the first health claim (hospital, physician, prescription) as the index date. Next, we identified a general population cohort excluding anyone with diagnosis codes for inflammatory bowel disease, multiple sclerosis or rheumatoid arthritis or use of any diseasemodifying therapies specific to multiple sclerosis, as these were part of the case definition for multiple sclerosis. ${ }^{8}$ See Appendix 1 for more details about selection of the study cohorts. Then, we selected controls using a uniform approach for each disease group, that is, matched on sex, year of birth (within 5 years before or after the birthdate of the corresponding case) and forward sortation area (based on postal code). We sought 5 controls for each case, but were unable to do so for every case. We assigned controls the index date of their matched cases.

\section{Comorbidities}

We applied validated case definitions developed in Manitoba to identify members of each cohort affected by any mood or anxiety disorder ( $\geq 1$ of depression, anxiety disorders, bipolar disorders), as well as by depression and anxiety disorders separately (Appendix 1, Tables e2 and e3). ${ }^{22} \mathrm{We}$ classified the date of the first claim for each condition as the diagnosis date. We identified physical comorbidity using the Aggregated Diagnosis Groups (major physical, not time-limited) of the Johns Hopkins Adjusted Clinical Group Case-Mix System. ${ }^{23-25}$

\section{Covariables}

We included the following matching factors and other covariables in the regression models: ${ }^{26}$ sex (male as reference group), age (updated annually), socioeconomic status at index date, region of residence at index date, physical comorbidity (updated annually), immune-mediated disease-specific procedures (ever) and use of disease-modifying therapy (updated annually). ${ }^{27-29}$

We categorized age as 18 to 24 years (reference group), 25 to 44 years, 45 to 64 years, and 65 years or older, reflecting the emphasis on immunizing individuals in the oldest age group.

To determine socioeconomic status, we linked postal code to census data at the level of dissemination area, and then calculated the Socioeconomic Factor Index version 2, which incorporates information about average household income, percent of single parent households, unemployment rate and high school education rate. For this index, scores less than 0 indicate higher socioeconomic status,${ }^{30}$ and missing values 
were imputed at the mean of 0 . We categorized socioeconomic status into quintiles (highest quintile as reference). We classified the region of residence as urban (Winnipeg, population > 600 000; Brandon, population > 47 000) or rural.

Physical comorbidity was based on Aggregated Diagnosis Group scores and categorized as 0 (reference group), 1, or 2 or above, with higher scores indicating greater comorbidity.

We included immune-mediated disease-specific procedures as a measure of disease severity. For inflammatory bowel disease, these included surgical procedures related to gastrointestinal resection or ostomy placement (Appendix 1, Table e4), and for rheumatoid arthritis, they included jointrelated surgical procedures (Appendix 1, Table e5); there were no relevant procedures for multiple sclerosis.

For inflammatory bowel disease and rheumatoid arthritis, we categorized immune therapies annually as none (reference group), any biologic (alone or in combination), or any antiinflammatory or traditional immunosuppressive therapy or corticosteroids. For multiple sclerosis, we categorized therapies as none, first-line or second-line (Appendix 1, Table e6).

\section{Influenza vaccination}

Annually, we identified influenza vaccinations using tariff codes 8791,8792 or 8799 , which are used by primary care providers, public health nurses and pharmacy providers.

\section{Statistical analysis}

We summarized the characteristics of the cohort using descriptive statistics, specifically means (with standard deviations), medians (with interquartile ranges) and frequencies (as percentages). We report the crude percentage of each cohort who had the influenza vaccination annually, along with a $95 \%$ confidence interval (CI) based on the binomial distribution. We also report percentages standardized by age and sex to the 2010 Canadian census population.

We accessed data for the period Apr. 1, 1984, to Mar. 31, 2016; however, for the regression analyses, we initially limited the study period to fiscal years 2006 to 2015 to reduce secular trends. We modelled the difference in the proportion of each cohort immunized annually so that we could estimate the absolute effects of cohort (immune-mediated disease v. matched controls) and psychiatric comorbidity on this outcome, as absolute effects are more useful than relative effects for policy-makers.

We used a binomial regression model with an identity link, as well as generalized estimating equations with an exchangeable correlation structure, accounting for differences in follow-up time by including the natural logarithm of person-years as the model offset. Covariables were those defined above. ${ }^{31}$

We tested for the presence of additive interaction effects between cohort and mood or anxiety disorders (entered as cohort $^{*}$ disorders), which we report as interaction contrasts (i.e., the difference of risk differences). A positive (synergistic or superadditive interaction) would indicate that the joint effects of cohort and mood and anxiety disorders exceeded the sum of their individual effects, whereas a negative (subadditive) interaction would indicate that the joint effects were less than the sum of the individual effects.

We report differences in terms of percentages and 95\% CIs. We repeated these analyses for each immunemediated disease cohort separately. In a complementary analysis, we further adjusted for the annual number of physician visits.

We conducted all statistical analyses using SAS software, version 9.4 (SAS Institute Inc.).

\section{Ethics approval}

The University of Manitoba Health Research Ethics Board approved the study. Manitoba's Health Information Privacy Committee approved data access.

\section{Results}

For the entire study period (fiscal years 1984-2015), we identified 32880 individuals with 1 or more immune-mediated inflammatory diseases (10 148 with inflammatory bowel disease, 6158 with multiple sclerosis and 16975 with rheumatoid arthritis), along with a total of 164152 matched controls (Table 1). The characteristics of these 3 disease cohorts were similar to those of the entire immune-mediated disease population (referred to hereafter as "cases") for the period 2006 to 2015. Cases and controls were well matched with respect to age, sex and socioeconomic status at the index date.

\section{Influenza vaccination}

The crude percentage of combined cases and controls who received influenza vaccination rose over time (Figure 1 and Appendix 1, Table e7). Moreover, the percentage of cases who received an influenza vaccination was similar across immune-mediated diseases after age and sex-standardization (Figure 2 and Appendix 1, Table e8).

In 2015 , the crude percentage of cases who received an influenza vaccination was $41.3 \%(n=8668$ of $20982 ; 95 \%$ CI $40.6 \%$ to $42.0 \%$ ), 7.6 percentage points more than matched controls $(n=35238$ of $104634 ; 33.7 \%, 95 \%$ CI $33.4 \%$ to $34.0 \%)$. Among cases, those with any mood or anxiety disorder had greater vaccine uptake than those without such a disorder $(43.7 \%, 95 \%$ CI $42.8 \%$ to $44.6 \%$ v. $38.3 \%$, $95 \%$ CI $37.3 \%$ to $39.3 \%)$. Similarly, among the controls, those with a mood or anxiety disorder had greater vaccine uptake than those without such a disorder $(37.5 \%, 95 \%$ CI $37.1 \%$ to $37.9 \%$ v. $30.5 \%$, $95 \%$ CI $30.1 \%$ to 30.9$)$. The percentage of people immunized increased with age and was highest among those aged 65 years or older (Appendix 1, Figure e1).

\section{Multivariable analysis}

On multivariable analysis for the period 2006 to 2015, cases with no mood or anxiety disorder had $6.44 \%$ (95\% CI 5.79 to 7.10) greater vaccination uptake than controls (Table 2). Among controls, having a mood or anxiety disorder was associated with $4.54 \%$ (95\% CI $4.20 \%$ to $4.89 \%$ ) more vaccination uptake. However, we observed a subadditive interaction between case and mood or anxiety disorder status $(-1.38 \%$, $95 \%$ CI $-2.26 \%$ to $-0.50 \%)$. Female as compared with male 


\begin{tabular}{|c|c|c|c|c|c|c|c|c|}
\hline \multirow[b]{3}{*}{ Characteristic } & \multicolumn{8}{|c|}{ Disease and cohort; no (\%) of patients* } \\
\hline & \multicolumn{2}{|c|}{ Inflammatory bowel disease } & \multicolumn{2}{|c|}{ Multiple sclerosis } & \multicolumn{2}{|c|}{ Rheumatoid arthritis } & \multicolumn{2}{|c|}{ IMID† } \\
\hline & $\begin{array}{c}\text { Controls } \\
n=50704\end{array}$ & $\begin{array}{c}\text { Cases } \\
n=10148\end{array}$ & $\begin{array}{c}\text { Controls } \\
n=30690\end{array}$ & $\begin{array}{c}\text { Cases† } \\
n=6158\end{array}$ & $\begin{array}{c}\text { Controls } \\
n=84756\end{array}$ & $\begin{array}{c}\text { Cases } \\
n=16975\end{array}$ & $\begin{array}{c}\text { Controls } \\
n=164152\end{array}$ & $\begin{array}{c}\text { Cases } \dagger \\
n=32880\end{array}$ \\
\hline \multicolumn{9}{|c|}{ Whole population, 1984-2015 } \\
\hline Sex, female & $\begin{array}{c}27663 \\
(54.6)\end{array}$ & $\begin{array}{l}5536 \\
(54.6)\end{array}$ & $\begin{array}{l}21564 \\
(70.3)\end{array}$ & $\begin{array}{l}4322 \\
(70.2)\end{array}$ & $\begin{array}{l}61231 \\
(72.2)\end{array}$ & $\begin{array}{l}12263 \\
(72.2)\end{array}$ & $\begin{array}{c}109065 \\
(66.4)\end{array}$ & $\begin{array}{r}21841 \\
(66.4)\end{array}$ \\
\hline $\begin{array}{l}\text { Age at diagnosis, yr, } \\
\text { mean } \pm \text { SD (range) }\end{array}$ & $\begin{array}{c}41.7 \pm 17.0 \\
(18 \text { to } 99)\end{array}$ & $\begin{array}{l}41.7 \pm 17.0 \\
(18 \text { to } 99)\end{array}$ & $\begin{array}{c}42.1 \pm 13.5 \\
(18 \text { to } 97)\end{array}$ & $\begin{array}{l}42.1 \pm 13.5 \\
(18 \text { to } 96)\end{array}$ & $\begin{array}{l}54.0 \pm 16.1 \\
(18 \text { to } 105)\end{array}$ & $\begin{array}{l}54.0 \pm 16.1 \\
(18 \text { to } 103)\end{array}$ & $\begin{array}{l}48.0 \pm 17.1 \\
(18 \text { to } 105)\end{array}$ & $\begin{array}{l}48.0 \pm 17.1 \\
(18 \text { to } 103)\end{array}$ \\
\hline $\begin{array}{l}\text { Duration of follow-up } \\
\text { from index date, yr, } \\
\text { median (IQR) }\end{array}$ & $\begin{array}{c}11.9 \\
(4.92 \text { to } 21.8)\end{array}$ & $\begin{array}{c}13.3 \\
(5.93 \text { to } 22.8)\end{array}$ & $\begin{array}{c}13.9 \\
(5.91 \text { to } 23.6)\end{array}$ & $\begin{array}{c}13.9 \\
(6.28 \text { to } 22.5)\end{array}$ & $\begin{array}{c}11.1 \\
\text { (4.93 to } 19.3)\end{array}$ & $\begin{array}{c}11.4 \\
(5.51 \text { to } 19.0)\end{array}$ & $\begin{array}{c}11.9 \\
\text { (5.12 to } 20.8)\end{array}$ & $\begin{array}{c}12.4 \\
\text { (5.79 to } 20.7)\end{array}$ \\
\hline $\begin{array}{l}\text { Urban region of } \\
\text { residence }\end{array}$ & $\begin{array}{c}33799 \\
(66.7)\end{array}$ & $\begin{array}{c}6763 \\
(66.6)\end{array}$ & $\begin{array}{c}20685 \\
(67.4)\end{array}$ & $\begin{array}{l}4154 \\
(67.5)\end{array}$ & $\begin{array}{l}50281 \\
(59.3)\end{array}$ & $\begin{array}{l}10070 \\
(59.3)\end{array}$ & $\begin{array}{c}103468 \\
(63.0)\end{array}$ & $\begin{array}{r}20727 \\
(63.0)\end{array}$ \\
\hline $\begin{array}{l}\text { Socioeconomic } \\
\text { status, } \neq \S \text { mean } \pm \text { SD }\end{array}$ & $-0.23 \pm 0.88$ & $-0.26 \pm 0.91$ & $-0.22 \pm 0.88$ & $-0.25 \pm 0.91$ & $0.06 \pm 1.01$ & $0.03 \pm 1.03$ & $-0.08 \pm 0.96$ & $-0.11 \pm 0.99$ \\
\hline \multicolumn{9}{|c|}{ Physician visits in prior year } \\
\hline $0-4$ & $\begin{array}{c}41688 \\
(82.2)\end{array}$ & $\begin{array}{l}6569 \\
(64.7)\end{array}$ & $\begin{array}{c}24637 \\
(80.3)\end{array}$ & $\begin{array}{l}3860 \\
(62.7)\end{array}$ & $\begin{array}{l}63997 \\
(75.5)\end{array}$ & $\begin{array}{l}8416 \\
(45.6)\end{array}$ & $\begin{array}{c}128760 \\
(78.4)\end{array}$ & $\begin{array}{l}19997 \\
(60.8)\end{array}$ \\
\hline $5-9$ & $\begin{array}{l}6517 \\
(12.9)\end{array}$ & $\begin{array}{l}2172 \\
(21.4)\end{array}$ & $\begin{array}{c}4196 \\
(13.7)\end{array}$ & $\begin{array}{l}1313 \\
(21.3)\end{array}$ & $\begin{array}{l}14230 \\
(16.8)\end{array}$ & $\begin{array}{l}3718 \\
(21.9)\end{array}$ & $\begin{array}{c}24623 \\
(15.0)\end{array}$ & $\begin{array}{l}7297 \\
(22.2)\end{array}$ \\
\hline$\geq 10$ & $\begin{array}{l}2499 \\
(4.9)\end{array}$ & $\begin{array}{c}1407 \\
(13.9)\end{array}$ & $\begin{array}{l}1857 \\
(6.0)\end{array}$ & $\begin{array}{c}985 \\
(16.0)\end{array}$ & $\begin{array}{l}6529 \\
(7.7)\end{array}$ & $\begin{array}{l}4841 \\
(28.5)\end{array}$ & $\begin{array}{c}10769 \\
(6.6)\end{array}$ & $\begin{array}{l}5586 \\
(17.0)\end{array}$ \\
\hline \multicolumn{9}{|c|}{ Comorbidity status at study start } \\
\hline \multicolumn{9}{|l|}{ No. of ADGs } \\
\hline 0 & $\begin{array}{c}42318 \\
(83.5)\end{array}$ & $\begin{array}{c}6749 \\
(66.5)\end{array}$ & $\begin{array}{c}26373 \\
(85.9)\end{array}$ & $\begin{array}{l}4389 \\
(71.3)\end{array}$ & $\begin{array}{c}65883 \\
(77.7)\end{array}$ & $\begin{array}{l}11216 \\
(66.1)\end{array}$ & $\begin{array}{c}133038 \\
(81.0)\end{array}$ & $\begin{array}{c}22275 \\
(67.7)\end{array}$ \\
\hline 1 & $\begin{array}{l}7246 \\
(14.3)\end{array}$ & $\begin{array}{l}2789 \\
(27.5)\end{array}$ & $\begin{array}{c}3763 \\
(12.3)\end{array}$ & $\begin{array}{c}1392 \\
(22.6)\end{array}$ & $\begin{array}{l}15605 \\
(18.4)\end{array}$ & $\begin{array}{l}4643 \\
(27.4)\end{array}$ & $\begin{array}{r}26227 \\
(16.0)\end{array}$ & $\begin{array}{c}8561 \\
(26.0)\end{array}$ \\
\hline$\geq 2$ & $\begin{array}{l}1140 \\
(2.2)\end{array}$ & $\begin{array}{l}610 \\
(6.0)\end{array}$ & $\begin{array}{l}554 \\
(1.8)\end{array}$ & $\begin{array}{l}377 \\
(6.1)\end{array}$ & $\begin{array}{l}3268 \\
(3.9)\end{array}$ & $\begin{array}{l}1116 \\
(6.6)\end{array}$ & $\begin{array}{l}4887 \\
(3.0)\end{array}$ & $\begin{array}{l}2044 \\
(6.2)\end{array}$ \\
\hline $\begin{array}{l}\text { Any mood or anxiety } \\
\text { disorder }\end{array}$ & $\begin{array}{c}10979 \\
(21.7)\end{array}$ & $\begin{array}{l}3147 \\
(31.0)\end{array}$ & $\begin{array}{c}7431 \\
(24.2)\end{array}$ & $\begin{array}{l}2485 \\
(40.4)\end{array}$ & $\begin{array}{c}22078 \\
(26.0)\end{array}$ & $\begin{array}{l}5735 \\
(33.8)\end{array}$ & $\begin{array}{l}39911 \\
(24.3)\end{array}$ & $\begin{array}{l}11171 \\
(34.0)\end{array}$ \\
\hline Depression & $\begin{array}{c}9590 \\
(18.9)\end{array}$ & $\begin{array}{l}2764 \\
(27.2)\end{array}$ & $\begin{array}{l}6546 \\
(21.3) \\
\end{array}$ & $\begin{array}{l}2197 \\
(35.7) \\
\end{array}$ & $\begin{array}{l}18853 \\
(22.2)\end{array}$ & $\begin{array}{c}4864 \\
(28.7) \\
\end{array}$ & $\begin{array}{c}34480 \\
(21.0)\end{array}$ & $\begin{array}{l}9645 \\
(29.3) \\
\end{array}$ \\
\hline Anxiety disorder & $\begin{array}{c}13438 \\
(26.5)\end{array}$ & $\begin{array}{l}3453 \\
(34.0)\end{array}$ & $\begin{array}{l}8931 \\
(29.1)\end{array}$ & $\begin{array}{l}2378 \\
(38.6)\end{array}$ & $\begin{array}{c}25922 \\
(30.6)\end{array}$ & $\begin{array}{l}6074 \\
(35.8)\end{array}$ & $\begin{array}{c}47639 \\
(29.0)\end{array}$ & $\begin{array}{l}11707 \\
(35.6)\end{array}$ \\
\hline Bipolar disorder & $\begin{array}{l}1562 \\
(3.1)\end{array}$ & $\begin{array}{l}548 \\
(5.4)\end{array}$ & $\begin{array}{l}1151 \\
(3.8)\end{array}$ & $\begin{array}{l}368 \\
(6.0)\end{array}$ & $\begin{array}{l}2963 \\
(3.5)\end{array}$ & $\begin{array}{l}716 \\
(4.2)\end{array}$ & $\begin{array}{l}5583 \\
(3.4)\end{array}$ & $\begin{array}{l}1602 \\
(4.9)\end{array}$ \\
\hline Population, 2006-2015 & $n=40364$ & $n=8458$ & $n=24154$ & $n=4748$ & $n=64510$ & $n=12984$ & $n=127310$ & $n=25832$ \\
\hline Sex, female & $\begin{array}{c}22207 \\
(55.0)\end{array}$ & $\begin{array}{l}4623 \\
(54.7)\end{array}$ & $\begin{array}{c}17247 \\
(71.4)\end{array}$ & $\begin{array}{l}3399 \\
(71.6)\end{array}$ & $\begin{array}{c}47102 \\
(73.0)\end{array}$ & $\begin{array}{l}9458 \\
(72.8)\end{array}$ & $\begin{array}{c}85349 \\
(67.0)\end{array}$ & $\begin{array}{l}8602 \\
(33.3)\end{array}$ \\
\hline $\begin{array}{l}\text { Age at diagnosis, } y r \text {, } \\
\text { mean } \pm S D \text { (range) }\end{array}$ & $\begin{array}{c}41.2 \pm 16.2 \\
(18 \text { to } 99)\end{array}$ & $\begin{array}{c}41.0 \pm 16.2 \\
(18 \text { to } 99)\end{array}$ & $\begin{array}{c}40.9 \pm 12.2 \\
(18 \text { to } 97)\end{array}$ & $\begin{array}{l}40.2 \pm 11.9 \\
(18 \text { to } 94)\end{array}$ & $\begin{array}{l}51.8 \pm 15.2 \\
(18 \text { to } 105)\end{array}$ & $\begin{array}{l}51.4 \pm 15.4 \\
(18 \text { to } 102)\end{array}$ & $\begin{array}{l}46.4 \pm 16.0 \\
(18 \text { to } 105)\end{array}$ & $\begin{array}{l}45.9 \pm 16.0 \\
(18 \text { to } 102)\end{array}$ \\
\hline $\begin{array}{l}\text { Urban region of } \\
\text { residence }\end{array}$ & $\begin{array}{c}26558 \\
(65.8)\end{array}$ & $\begin{array}{c}5574 \\
(65.9)\end{array}$ & $\begin{array}{l}15925 \\
(65.9)\end{array}$ & $\begin{array}{l}3153 \\
(66.4)\end{array}$ & $\begin{array}{c}37559 \\
(58.2)\end{array}$ & $\begin{array}{c}7609 \\
(58.6)\end{array}$ & $\begin{array}{c}78949 \\
(62.0)\end{array}$ & $\begin{array}{l}16104 \\
(62.3)\end{array}$ \\
\hline $\begin{array}{l}\text { Socioeconomic status, } \neq \\
\text { mean } \pm \text { SD }\end{array}$ & $-0.22 \pm 0.85$ & $-0.26 \pm 0.89$ & $-0.21 \pm 0.85$ & $-0.25 \pm 0.89$ & $0.06 \pm 1.0$ & $0.04 \pm 1.0$ & $-0.08 \pm 0.95$ & $-0.11 \pm 0.98$ \\
\hline \multicolumn{9}{|c|}{ Physician visits in prior year } \\
\hline $0-4$ & $\begin{array}{c}26789 \\
(66.4)\end{array}$ & $\begin{array}{l}3810 \\
(45.0)\end{array}$ & $\begin{array}{l}15224 \\
(63.0)\end{array}$ & $\begin{array}{l}2260 \\
(47.6)\end{array}$ & $\begin{array}{c}37960 \\
(58.8)\end{array}$ & $\begin{array}{c}4745 \\
(36.5)\end{array}$ & $\begin{array}{l}78891 \\
(62.0)\end{array}$ & $\begin{array}{c}10716 \\
(41.5)\end{array}$ \\
\hline $5-9$ & $\begin{array}{l}8700 \\
(21.6)\end{array}$ & $\begin{array}{l}2358 \\
(27.9)\end{array}$ & $\begin{array}{l}5711 \\
(23.6)\end{array}$ & $\begin{array}{l}1313 \\
(27.7)\end{array}$ & $\begin{array}{c}16064 \\
(24.9)\end{array}$ & $\begin{array}{l}3593 \\
(27.7)\end{array}$ & $\begin{array}{c}30072 \\
(23.6)\end{array}$ & $\begin{array}{l}7171 \\
(27.8)\end{array}$ \\
\hline$\geq 10$ & $\begin{array}{l}4875 \\
(12.1)\end{array}$ & $\begin{array}{l}2290 \\
(27.1)\end{array}$ & $\begin{array}{l}3219 \\
(13.3)\end{array}$ & $\begin{array}{l}1175 \\
(24.7)\end{array}$ & $\begin{array}{l}10486 \\
(16.3)\end{array}$ & $\begin{array}{l}4646 \\
(35.8)\end{array}$ & $\begin{array}{l}18347 \\
(14.4)\end{array}$ & $\begin{array}{l}7945 \\
(30.8)\end{array}$ \\
\hline
\end{tabular}




\begin{tabular}{|c|c|c|c|c|c|c|c|c|}
\hline \multirow[b]{3}{*}{ Characteristic } & \multicolumn{8}{|c|}{ Disease and cohort; no (\%) of patients* } \\
\hline & \multicolumn{2}{|c|}{ Inflammatory bowel disease } & \multicolumn{2}{|c|}{ Multiple sclerosis } & \multicolumn{2}{|c|}{ Rheumatoid arthritis } & \multicolumn{2}{|c|}{ IMID† } \\
\hline & $\begin{array}{c}\text { Controls } \\
n=50704\end{array}$ & $\begin{array}{c}\text { Cases } † \\
n=10148\end{array}$ & $\begin{array}{c}\text { Controls } \\
n=30690\end{array}$ & $\begin{array}{c}\text { Cases† } \\
n=6158\end{array}$ & $\begin{array}{c}\text { Controls } \\
n=84756\end{array}$ & $\begin{array}{c}\text { Casest } \\
n=16975\end{array}$ & $\begin{array}{c}\text { Controls } \\
n=164152\end{array}$ & $\begin{array}{c}\text { Casest } \\
n=32880\end{array}$ \\
\hline \multicolumn{9}{|l|}{ No. of ADGs } \\
\hline 0 & $\begin{array}{c}33895 \\
(84.0)\end{array}$ & $\begin{array}{l}5546 \\
(65.6)\end{array}$ & $\begin{array}{c}20903 \\
(86.5)\end{array}$ & $\begin{array}{l}3371 \\
(71.0)\end{array}$ & $\begin{array}{c}51284 \\
(79.5)\end{array}$ & $\begin{array}{l}8594 \\
(66.2)\end{array}$ & $\begin{array}{c}104753 \\
(82.3)\end{array}$ & $\begin{array}{c}17434 \\
(67.5)\end{array}$ \\
\hline 1 & $\begin{array}{l}5638 \\
(14.0)\end{array}$ & $\begin{array}{l}2390 \\
(28.2)\end{array}$ & $\begin{array}{l}2892 \\
(12.0)\end{array}$ & $\begin{array}{l}1089 \\
(22.9)\end{array}$ & $\begin{array}{l}11140 \\
(17.3)\end{array}$ & $\begin{array}{l}3577 \\
(27.5)\end{array}$ & $\begin{array}{l}19340 \\
(15.2)\end{array}$ & $\begin{array}{l}6827 \\
(26.4)\end{array}$ \\
\hline Depression & $\begin{array}{c}7992 \\
(19.8)\end{array}$ & $\begin{array}{l}2343 \\
(27.7)\end{array}$ & $\begin{array}{l}5410 \\
(22.4)\end{array}$ & $\begin{array}{c}1742 \\
(36.7)\end{array}$ & $\begin{array}{l}14548 \\
(22.6)\end{array}$ & $\begin{array}{l}3808 \\
(29.3)\end{array}$ & $\begin{array}{c}27517 \\
(21.6)\end{array}$ & $\begin{array}{c}7723 \\
(29.9)\end{array}$ \\
\hline Anxiety disorder & $\begin{array}{l}11444 \\
(28.4)\end{array}$ & $\begin{array}{l}2980 \\
(35.2)\end{array}$ & $\begin{array}{l}7543 \\
(31.2)\end{array}$ & $\begin{array}{c}1983 \\
(41.8)\end{array}$ & $\begin{array}{c}21332 \\
(33.1)\end{array}$ & $\begin{array}{l}5063 \\
(39.0)\end{array}$ & $\begin{array}{c}39735 \\
(31.2)\end{array}$ & $\begin{array}{l}9839 \\
(38.1)\end{array}$ \\
\hline Bipolar disorder & $\begin{array}{l}1326 \\
(3.3)\end{array}$ & $\begin{array}{c}465 \\
(5.5)\end{array}$ & $\begin{array}{l}968 \\
(4.0)\end{array}$ & $\begin{array}{c}301 \\
(6.3)\end{array}$ & $\begin{array}{l}2347 \\
(3.6)\end{array}$ & $\begin{array}{l}575 \\
(4.4)\end{array}$ & $\begin{array}{l}4559 \\
(3.6)\end{array}$ & $\begin{array}{l}1312 \\
(5.1)\end{array}$ \\
\hline \multicolumn{9}{|c|}{$\begin{array}{l}\text { Note: IMID = immune-mediated inflammatory disease (IMID cohort combines patients with inflammatory bowel disease, multiple sclerosis and rheumatoid arthritis). } \\
\text { "Except where indicated otherwise. } \\
\text { †A small number of individuals met the case definitions for more than } 1 \text { of the IMIDs of interest. These were included in the analysis, to ensure generalizability of the } \\
\text { findings, but they were counted only once in the combined IMID cohort. In this situation, they were classified on the basis of the IMID with the earliest index date in the } \\
\text { coverage period. Some cases did not have } 5 \text { matched controls (IMID, } n=90 \text {; inflammatory bowel disease, } n=14 \text {; multiple sclerosis, } n=36 \text {; rheumatoid arthritis, } n=42 \text { ). } \\
\text { fSocioeconomic status is reported as the Socioeconomic Factor Index score, incorporating information about average household income, percent of single-parent } \\
\text { households, unemployment rate and high school education rate, with missing values imputed at the mean of } 0 \text {. With this measure, values less than } 0 \text { indicate higher } \\
\text { socioeconomic status. } \\
\text { \$Missing data: for multiple sclerosis, } 20 \text {; for multiple sclerosis controls, } 110 \text {; for inflammatory bowel disease, } 21 \text {; for inflammatory bowel disease controls, } 76 \text {; for rheumatoid } \\
\text { arthritis, 40; for rheumatoid arthritis controls, } 160 \text {; imputed at population mean }=0 \text {. }\end{array}$} \\
\hline
\end{tabular}

sex, older age, living in an urban not rural region, more physical comorbidities, prior disease-specific surgery and use of immune therapies were all associated with increased vaccine uptake. Our findings were similar after further adjustment for physician visits (Appendix 1, Table e9).

When we conducted separate analyses for depression and anxiety disorders, the findings were similar to those for mood and anxiety disorders combined (Table 3 and Table 4). When we conducted analyses for individual immunemediated diseases, findings in the rheumatoid arthritis cohort were similar to those for the combined immunemediated disease cohort. Findings in the inflammatory bowel disease cohort were similar in magnitude and direction to those in the combined immune-mediated disease cohort but were not statistically significant. In contrast, we did not observe a departure from additivity in the multiple sclerosis cohort for any mood or anxiety disorder, but we did observe a superadditive effect for depression and anxiety disorders (Tables 2, 3 and 4).

\section{Interpretation}

In this population-based study, vaccination uptake increased over the 30-year study period in 3 immune-mediated disease cohorts and in matched cohorts without immune-mediated disease, in concert with programmatic changes. In 1999, vaccinations became publicly funded for Manitobans aged 65 years and older, those with some chronic conditions and health care workers. In 2005, vaccination funding was extended to those with chronic respiratory disease. However, despite vaccination coverage for the entire provincial population as of 2010 and access through pharmacists as of 2014, vaccination rates in all populations remain lower than desired. In 2015, only 4 in 10 persons with immune-mediated disease were vaccinated annually, which was only slightly better than the 3 in 10 persons without immune-mediated disease who were vaccinated annually. In the matched population, having a mood or anxiety disorder was associated with increased vaccination uptake. However, the joint effect of an immunemediated disease and mood or anxiety disorder on increasing uptake was subadditive.

In Canada, the target vaccination rate for high-risk populations and adults aged 65 years or older is $80 \%$; vaccination is also recommended in immune-mediated disease guidelines. ${ }^{32-34} \mathrm{We}$ found vaccination rates well below this target. In the 2013/14 Canadian Community Health Survey, only $37.8 \%$ of adults aged 18 to 64 years of age with a chronic medical condition were vaccinated. ${ }^{35}$

Findings from prior studies involving persons with inflammatory bowel disease, multiple sclerosis and rheumatoid arthritis 


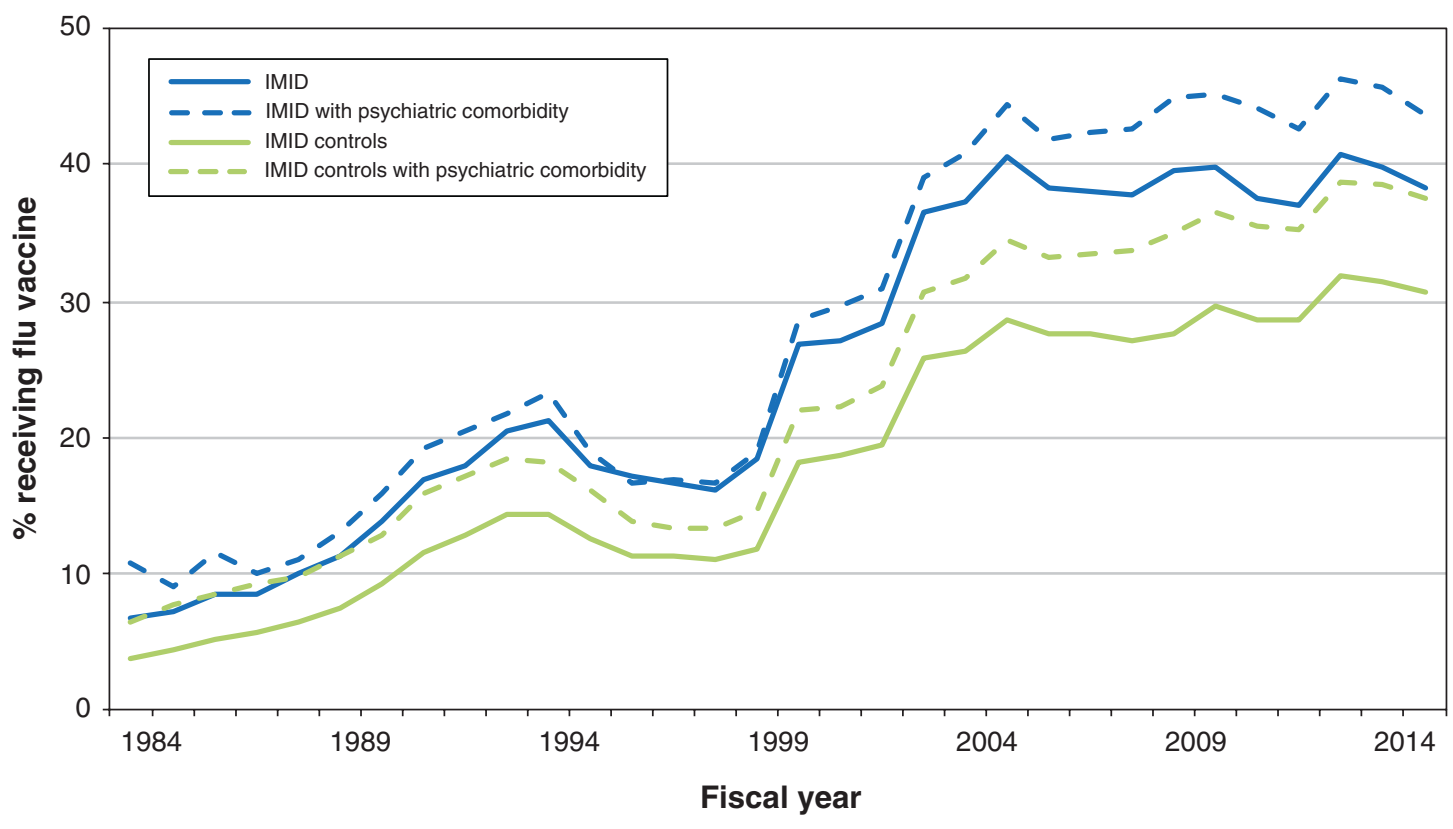

Figure 1: Percentage of persons in the combined immune-mediated inflammatory disease (IMID) cohort and the combined cohort of matched controls who received an influenza vaccination, stratified by psychiatric comorbidity status, fiscal years 1984 to 2015 (i.e., Apr. 1, 1984 , to Mar. 31, 2016).

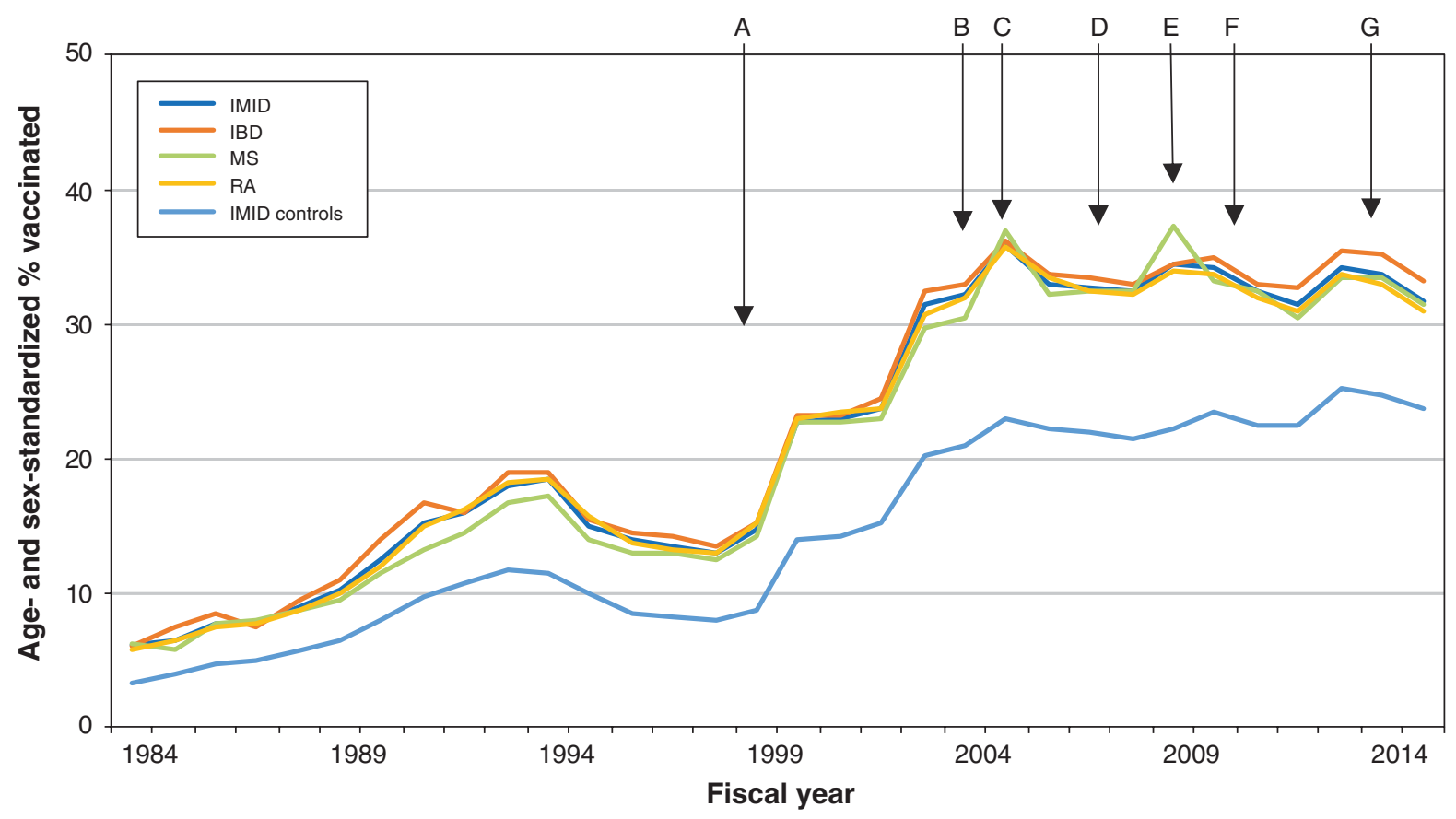

Figure 2: Age- and sex-standardized percentage of persons in the combined immune-mediated inflammatory disease (IMID) cohort, the individual disease cohorts and the combined cohort of matched controls who received an influenza vaccination, fiscal years 1984 to 2015 (i.e., Apr. 1 , 1984, to Mar. 31, 2016). Arrows indicate changes in groups eligible for provincial (public) funding of vaccination: in 1999 , age $\geq 65$ years, chronic conditions, health care workers (A); in 2004, children aged 6-23 months and their families (B); in 2005, patients with chronic respiratory diseases (C); in 2007, pregnant women (D); in 2009, H1N1 epidemic (E); in 2010, coverage for entire provincial population (F); and in 2014, pharmacists able to administer influenza vaccine $(G)$. Note: IBD = inflammatory bowel disease, $M S=$ multiple sclerosis, RA $=$ rheumatoid arthritis. 
Table 2: Multivariable adjusted risk differences for the association of immune-mediated disease, any mood or anxiety disorder and uptake of influenza vaccination

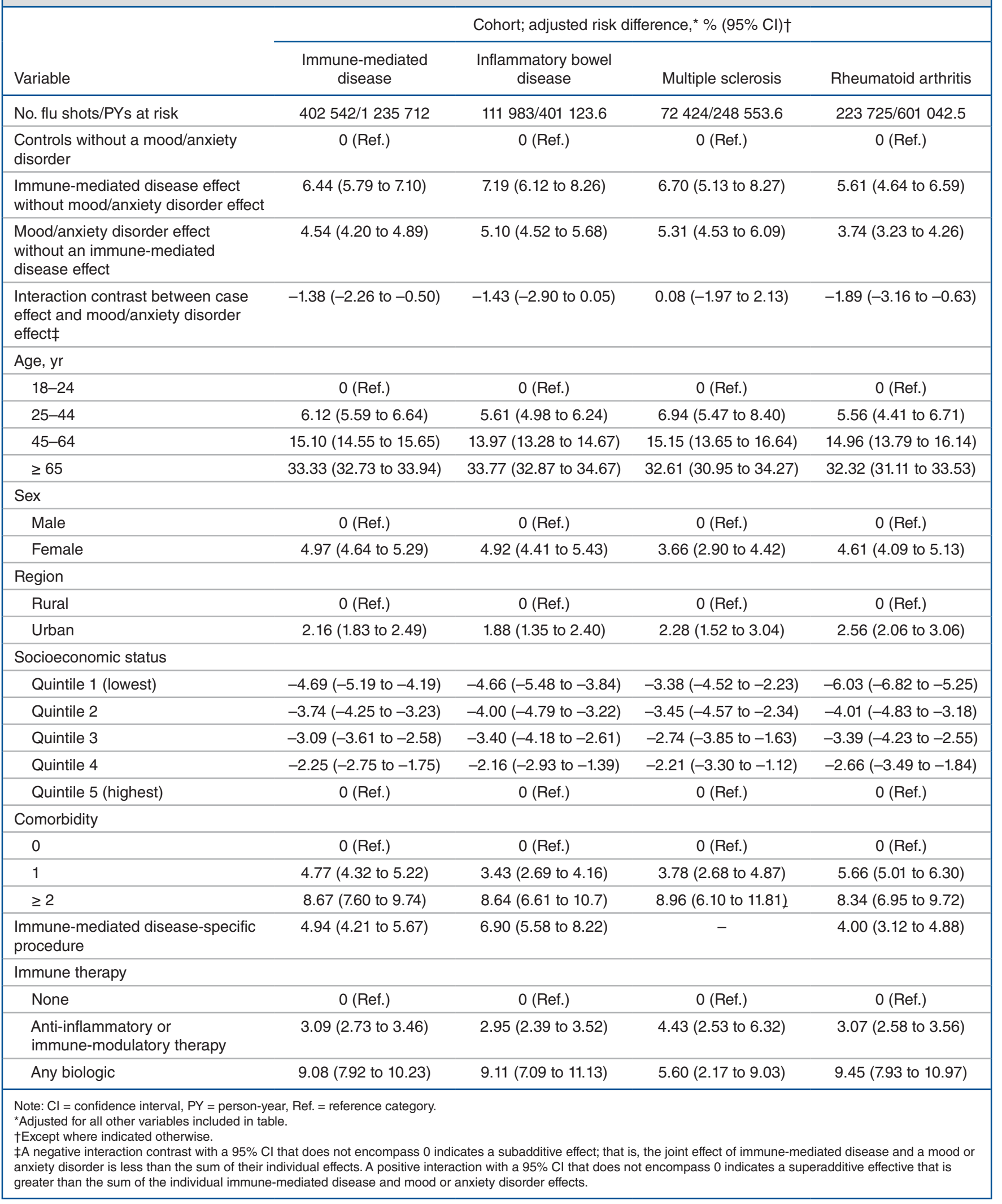


Table 3: Multivariable adjusted risk differences for the association of immune-mediated inflammatory disease, depressive disorder and uptake of influenza vaccination

\begin{tabular}{|c|c|c|c|c|}
\hline \multirow[b]{2}{*}{ Variable } & \multicolumn{4}{|c|}{ Cohort; adjusted risk difference, ${ }^{*} \%(95 \% \mathrm{Cl}) \dagger$} \\
\hline & $\begin{array}{l}\text { Immune-mediated } \\
\text { disease }\end{array}$ & $\begin{array}{l}\text { Inflammatory bowel } \\
\text { disease }\end{array}$ & Multiple sclerosis & Rheumatoid arthritis \\
\hline No. flu shots/PYs at risk & 402 542/1 235712 & $111983 / 401123.6$ & 72 424/248 553.6 & 223 725/601 042.5 \\
\hline Controls without depression & 0 (Ref.) & 0 (Ref.) & 0 (Ref.) & 0 (Ref.) \\
\hline $\begin{array}{l}\text { Immune-mediated disease effect } \\
\text { without depression effect }\end{array}$ & 6.08 (5.49 to 6.66$)$ & $6.81(5.82$ to 7.80$)$ & $5.81(4.45$ to 7.18$)$ & 5.52 (4.65 to 6.38$)$ \\
\hline $\begin{array}{l}\text { Depression effect without immune- } \\
\text { mediated disease effect }\end{array}$ & 4.15 (3.75 to 4.56$)$ & 5.11 (4.40 to 5.82$)$ & 4.28 (3.39 to 5.17$)$ & 3.41 (2.82 to 4.00$)$ \\
\hline $\begin{array}{l}\text { Interaction contrast between case } \\
\text { effect and depressive disorder } \\
\text { effectł }\end{array}$ & $-0.98(-1.93$ to -0.04$)$ & $-1.03(-2.70$ to 0.64$)$ & $2.43(0.36$ to 4.49$)$ & $-2.53(-3.88$ to -1.18$)$ \\
\hline \multicolumn{5}{|l|}{ Age, yr } \\
\hline $18-24$ & 0 (Ref.) & 0 (Ref.) & 0 (Ref.) & 0 (Ref.) \\
\hline $25-44$ & 6.36 (5.83 to 6.89$)$ & 5.87 (5.24 to 6.51$)$ & 7.33 (5.90 to 8.77$)$ & 5.74 (4.58 to 6.90$)$ \\
\hline $45-64$ & $15.45(14.90$ to 16.00$)$ & $14.36(13.66$ to 15.05$)$ & 15.65 (14.19 to 17.11$)$ & $15.22(14.04$ to 16.40$)$ \\
\hline$\geq 65$ & 33.75 (33.14 to 34.35$)$ & 34.26 (33.36 to 35.15$)$ & 33.22 (31.59 to 34.86$)$ & 32.60 (31.39 to 33.82$)$ \\
\hline \multicolumn{5}{|l|}{ Sex } \\
\hline Male & 0 (Ref.) & 0 (Ref.) & 0 (Ref.) & 0 (Ref.) \\
\hline Female & 5.19 (4.87 to 5.52$)$ & 5.12 (4.62 to 5.63$)$ & 3.96 (3.21 to 4.72 ) & 4.80 (4.28 to 5.32$)$ \\
\hline \multicolumn{5}{|l|}{ Region } \\
\hline Rural & 0 (Ref.) & 0 (Ref.) & 0 (Ref.) & 0 (Ref.) \\
\hline Urban & 2.28 (1.95 to 2.61$)$ & 1.99 (1.47 to 2.52$)$ & 2.47 (1.71 to 3.23$)$ & 2.67 (2.17 to 3.16$)$ \\
\hline \multicolumn{5}{|l|}{ Socioeconomic status } \\
\hline Quintile 1 (lowest) & $-4.71(-5.21$ to -4.20$)$ & $-4.69(-5.51$ to -3.87$)$ & $-3.41(-4.56$ to -2.27$)$ & $-6.03(-6.82$ to -5.25$)$ \\
\hline Quintile 2 & $-3.81(-4.32$ to -3.29$)$ & $-4.15(-4.94$ to -3.36$)$ & $-3.57(-4.58$ to -2.45$)$ & $-4.02(-4.85$ to -3.19$)$ \\
\hline Quintile 3 & $-3.14(-3.65$ to -2.62$)$ & $-3.47(-4.26$ to -2.68$)$ & $-2.83(-3.94$ to -1.72$)$ & $-3.40(-4.24$ to -2.56$)$ \\
\hline Quintile 4 & $-2.27(-2.77$ to -1.77$)$ & $-2.19(-2.97$ to -0.64$)$ & $-2.29(-3.38$ to -1.19$)$ & $-2.66(-3.48$ to -1.84$)$ \\
\hline Quintile 5 (highest) & 0 (Ref.) & 0 (Ref.) & 0 (Ref.) & 0 (Ref.) \\
\hline \multicolumn{5}{|l|}{ Comorbidity } \\
\hline 0 & 0 (Ref.) & 0 (Ref.) & 0 (Ref.) & 0 (Ref.) \\
\hline 1 & 4.86 (4.41 to 5.31$)$ & 3.53 (2.80 to 4.27$)$ & 3.89 (2.79 to 4.98$)$ & 5.73 (5.09 to 6.37 ) \\
\hline$\geq 2$ & 8.73 (7.65 to 9.80$)$ & 8.65 (6.62 to 10.68$)$ & 9.04 (6.18 to 11.90$)$ & 8.41 (7.02 to 9.79$)$ \\
\hline $\begin{array}{l}\text { Immune-mediated disease-specific } \\
\text { procedure }\end{array}$ & 4.96 (4.23 to 5.69$)$ & 6.95 (5.63 to 8.27$)$ & - & 4.01 (3.13 to 4.89 ) \\
\hline \multicolumn{5}{|l|}{ Immune therapy } \\
\hline None & 0 (Ref.) & 0 (Ref.) & 0 (Ref.) & 0 (Ref.) \\
\hline $\begin{array}{l}\text { Anti-inflammatory or immune- } \\
\text { modulatory therapy }\end{array}$ & 3.10 (2.74 to 3.47$)$ & 2.97 (2.40 to 3.54 ) & 4.40 (2.51 to 6.29$)$ & 3.06 (2.57 to 3.55$)$ \\
\hline Any biologic & 9.08 (7.92 to 10.23$)$ & 9.08 (7.06 to 11.10$)$ & 5.54 (2.11 to 8.96$)$ & 9.46 (7.94 to 10.98$)$ \\
\hline \multicolumn{5}{|c|}{$\begin{array}{l}\text { Note: } \mathrm{Cl}=\text { confidence interval, } \mathrm{PY}=\text { person-year, Ref. = reference category. } \\
\text { *Adjusted for all other variables included in table. } \\
\text { †Except where indicated otherwise. } \\
\text { †A negative interaction contrast with a } 95 \% \mathrm{Cl} \text { that does not encompass } 0 \text { indicates a subadditive effect; that is, the joint effect of immune-mediated disease and depressive } \\
\text { disorder is less than the sum of their individual effects. A positive interaction with a } 95 \% \mathrm{Cl} \text { that does not encompass } 0 \text { indicates a superadditive effective that is greater } \\
\text { than the sum of the individual immune-mediated disease and depressive disorder effects. }\end{array}$} \\
\hline
\end{tabular}


Table 4: Multivariable adjusted risk differences for the association of immune-mediated inflammatory disease, anxiety disorder and uptake of influenza vaccination

\begin{tabular}{|c|c|c|c|c|}
\hline \multirow[b]{2}{*}{ Variable } & \multicolumn{4}{|c|}{ Cohort; adjusted risk difference, ${ }^{*} \%(95 \% \mathrm{Cl}) \dagger$} \\
\hline & $\begin{array}{l}\text { Immune-mediated } \\
\text { disease }\end{array}$ & $\begin{array}{l}\text { Inflammatory bowel } \\
\text { disease }\end{array}$ & Multiple sclerosis & Rheumatoid arthritis \\
\hline No. flu shots/PYs at risk & 402 542/1 235712 & $111983 / 401123.6$ & $72424 / 248553.6$ & 223 725/601 042.5 \\
\hline $\begin{array}{l}\text { Controls without anxiety } \\
\text { disorder }\end{array}$ & 0 (Ref.) & 0 (Ref.) & 0 (Ref.) & 0 (Ref.) \\
\hline $\begin{array}{l}\text { Immune-mediated disease } \\
\text { effect without anxiety disorder } \\
\text { effect }\end{array}$ & $6.58(5.96$ to 7.19$)$ & $6.89(5.86$ to 7.91$)$ & $7.78(6.35$ to 9.21$)$ & $5.72(4.80$ to 6.64$)$ \\
\hline $\begin{array}{l}\text { Anxiety effect without immune- } \\
\text { mediated disease effect }\end{array}$ & 4.41 (4.05 to 4.77$)$ & 4.93 (4.32 to 5.53$)$ & 4.95 (4.65 to 5.75$)$ & 3.75 (3.22 to 4.27 ) \\
\hline $\begin{array}{l}\text { Interaction contrast between } \\
\text { case effect and anxiety disorder } \\
\text { effectł }\end{array}$ & $-1.74(-2.62$ to -0.86$)$ & $-0.78(-2.27$ to 0.71$)$ & $2.43(0.36$ to 4.49$)$ & $-2.35(-3.62$ to -1.07$)$ \\
\hline \multicolumn{5}{|l|}{ Age, yr } \\
\hline $18-24$ & 0 (Ref.) & 0 (Ref.) & 0 (Ref.) & 0 (Ref.) \\
\hline $25-44$ & $6.22(5.70$ to 6.74$)$ & 5.72 (5.09 to 6.35$)$ & 7.12 (5.67 to 8.58$)$ & $5.62(4.47$ to 6.77$)$ \\
\hline $45-64$ & $15.21(14.67$ to 15.76$)$ & $14.08(13.39$ to 14.78$)$ & $15.36(13.88$ to 16.85$)$ & $15.03(13.86$ to 16.20$)$ \\
\hline$\geq 65$ & 33.44 (32.83 to 34.04$)$ & 33.88 (32.98 to 34.77$)$ & 32.83 (31.17 to 34.48$)$ & 32.38 (31.18 to 33.59$)$ \\
\hline \multicolumn{5}{|l|}{ Sex } \\
\hline Male & 0 (Ref.) & 0 (Ref.) & 0 (Ref.) & 0 (Ref.) \\
\hline Female & 5.10 (4.78 to 5.42$)$ & 5.06 (4.55 to 5.57$)$ & 3.86 (3.10 to 4.62 ) & $4.69(4.17$ to 5.21$)$ \\
\hline \multicolumn{5}{|l|}{ Region } \\
\hline Rural & 0 (Ref.) & 0 (Ref.) & 0 (Ref.) & 0 (Ref.) \\
\hline Urban & 2.20 (1.87 to 2.53$)$ & 1.91 (1.38 to 2.43 ) & 2.32 (1.56 to 3.09$)$ & 2.59 (2.09 to 3.09$)$ \\
\hline \multicolumn{5}{|l|}{ Socioeconomic status } \\
\hline Quintile 1 (lowest) & $-4.70(-5.21$ to -4.20$)$ & $-4.69(-5.51$ to -3.87$)$ & $-3.30(-4.44$ to -2.15$)$ & $-6.05(-6.84$ to -5.27$)$ \\
\hline Quintile 2 & $-3.73(-4.24$ to -3.22$)$ & $-4.04(-4.82$ to -3.25$)$ & $-3.40(-4.52$ to -2.29$)$ & $-4.00(-4.82$ to -3.17$)$ \\
\hline Quintile 3 & $-3.06(-3.57$ to -2.54$)$ & $-3.35(-4.14$ to -2.57$)$ & $-2.70(-3.81$ to -1.58$)$ & $-3.37(-4.20$ to -2.53$)$ \\
\hline Quintile 4 & $-2.24(-2.75$ to -1.74$)$ & $-2.15(-2.92$ to -1.38$)$ & $-2.16(-3.52$ to -1.06$)$ & $-2.67(-3.49$ to -1.84$)$ \\
\hline Quintile 5 (highest) & 0 (Ref.) & 0 (Ref.) & 0 (Ref.) & 0 (Ref.) \\
\hline \multicolumn{5}{|l|}{ Comorbidity } \\
\hline 0 & 0 (Ref.) & 0 (Ref.) & 0 (Ref.) & 0 (Ref.) \\
\hline 1 & 4.81 (4.36 to 5.26 ) & 3.46 (2.72 to 4.20$)$ & 3.86 (2.76 to 4.96$)$ & 5.68 (5.04 to 6.33$)$ \\
\hline$\geq 2$ & 8.75 (7.68 to 9.83 ) & $8.68(6.65$ to 10.71$)$ & 9.17 (6.31 to 12.04$)$ & 8.40 (7.02 to 9.79$)$ \\
\hline $\begin{array}{l}\text { Immune-mediated disease- } \\
\text { specific procedure }\end{array}$ & 4.98 (4.26 to 5.71$)$ & 6.91 (5.59 to 8.23$)$ & - & 4.04 (3.17 to 4.92 ) \\
\hline \multicolumn{5}{|l|}{ Immune therapy } \\
\hline None & 0 (Ref.) & 0 (Ref.) & 0 (Ref.) & 0 (Ref.) \\
\hline $\begin{array}{l}\text { Anti-inflammatory/ } \\
\text { immune-modulatory } \\
\text { therapy }\end{array}$ & 3.09 (2.72 to 3.45$)$ & 2.97 (2.40 to 3.53 ) & 4.48 (2.58 to 6.37$)$ & 3.06 (2.57 to 3.55$)$ \\
\hline Any biologic & 9.10 (7.95 to 10.30$)$ & 9.17 (7.15 to 11.20$)$ & 5.77 (2.31 to 9.24$)$ & 9.46 (7.93 to 10.98$)$ \\
\hline \multicolumn{5}{|c|}{$\begin{array}{l}\text { Note: } \mathrm{Cl}=\text { confidence interval, } \mathrm{PY}=\text { person-years, Ref. = reference category. } \\
\text { *Adjusted for all other variables included in table. } \\
\text { tExcept where indicated otherwise. } \\
\text { fA negative interaction contrast with a } 95 \% \mathrm{Cl} \text { that does not encompass } 0 \text { indicates a subadditive effect; that is, the joint effect of immune-mediated disease and anxiety } \\
\text { disorder is less than the sum of their individual effects. A positive interaction with a } 95 \% \mathrm{Cl} \text { that does not encompass } 0 \text { indicates a superadditive effective that is greater } \\
\text { than the sum of the individual immune-mediated disease and anxiety disorder effects. }\end{array}$} \\
\hline
\end{tabular}


have been inconsistent. Reported vaccination rates among those with inflammatory bowel disease have ranged from $6 \%$ to $80 \%{ }^{2,3}$ Investigation of temporal changes in vaccine uptake has been limited. In Israel, $16.1 \%$ of persons with Crohn disease were vaccinated in 2006; this increased to $38.3 \%$ by 2012. ${ }^{36}$ Findings were similar among persons with ulcerative colitis. $^{37}$ Reported uptake of influenza vaccine has also ranged widely among persons with rheumatoid arthritis, ${ }^{5,38-40}$ from $26.6 \%$ in a German outpatient clinic ${ }^{40}$ to $85 \%$ in specialty rheumatology centres in the United Kingdom. ${ }^{5}$ Comparable findings for multiple sclerosis are more limited. In Israel, $37.6 \%$ of 101 participants with multiple sclerosis reported receiving the seasonal influenza vaccine during the winter of 2009/10. ${ }^{4}$ In Norway, $60.7 \%$ of persons with multiple sclerosis received the pandemic (H1N1) vaccine in 2009/10. ${ }^{41}$

We observed a subadditive interaction between immunemediated disease and psychiatric comorbidity on vaccination uptake in the combined cohort and in the cohorts of persons with inflammatory bowel disease and rheumatoid arthritis. This finding may reflect competing demands during physician visits ${ }^{42}$ or reduced adherence to treatment recommendations by persons with psychiatric disorders, whether pharmacologic ${ }^{43-45}$ or health behaviours. ${ }^{46}$ However, we did not observe a subadditive interaction in the multiple sclerosis cohort, which may reflect differences in provincial programs of care for immune-mediated disease, which are more centralized for multiple sclerosis.

Older age, female sex, higher socioeconomic status, urban residence, greater disease severity (as evidenced by prior surgeries), physical comorbidities and use of immune therapies were associated with greater uptake of vaccination. These findings are consistent with those for the Canadian general population ${ }^{35}$ and earlier studies in immunemediated disease populations. ${ }^{3,36,37}$

Factors contributing to low vaccination rates include a lack of perceived susceptibility to influenza, lack of perceived severity of infection and lack of belief in the vaccine's effectiveness. ${ }^{2,3,47-49}$ Interventions to improve vaccination rates in populations of persons with immune-mediated disease have been tested. Use of electronic medical record alerts improved vaccination rates in immunosuppressed people seen in rheumatology clinics (outpatient, hospital-based); having a nurseled process improved vaccination rates further. ${ }^{50}$ At an inflammatory bowel disease clinic, distributing a vaccine questionnaire before attendance at the clinic and offering recommended vaccinations increased influenza vaccine uptake from $54 \%$ to $81 \%{ }^{51}$ Future studies aimed at improving implementation of such strategies are needed.

\section{Limitations}

Strengths of this study included the population-based design, application of validated case definitions for immune-mediated diseases and psychiatric comorbidity, and the extended study period. The female predominance and age distribution of our cohorts are consistent with the epidemiology of the diseases studied. ${ }^{52-54}$

Conduct of the study in a single province was a limitation. However, in the 2013/14 Canadian Community Health
Survey, the proportion of individuals vaccinated was similar across provinces, apart from Nova Scotia, Quebec and Newfoundland and Labrador. ${ }^{55}$ As such, our findings are likely generalizable within Canada, although they may not be generalizable elsewhere.

We may not have identified all vaccinations administered, given that vaccinations in private workplaces would not be captured in our data; however, underascertainment was likely to be nondifferential among the cohorts and would not fully account for our findings. Reassuringly, the percentage of Manitobans reporting influenza vaccinations in the Canadian Community Health Survey (30\%) was consistent with the percentage among our controls. ${ }^{55}$

We lacked clinical characteristics related to the immunemediated diseases, but we included some measures of disease severity and treatment status.

\section{Conclusion}

Uptake of influenza vaccination was lower than desired in populations with immune-mediated disease. Given the increased susceptibility of these populations to influenza and related complications, it is essential that action be taken to ameliorate this gap in preventive care. Although having a mood or anxiety disorder was associated with increased influenza vaccine uptake among Manitobans without immunemediated disease, comorbid mood or anxiety disorder interacted negatively with immune-mediated disease. This suggests that the association of psychiatric comorbidity with other preventive health behaviours should also be evaluated in people with immune-mediated diseases.

\section{References}

1. Blumentals WA, Arreglado A, Napalkov P, et al. Rheumatoid arthritis and the incidence of influenza and influenza-related complications: a retrospective cohort study. BMC Musculoskelet Disord 2012;13:158.

2. Waszczuk K, Waszczuk E, Szenborn L. Can we better protect patients with inflammatory bowel disease against infections - patient attitude and personal immunization knowledge. Acta Gastroenterol Belg 2018;81:257-61.

3. Wasan SK, Calderwood AH, Long MD, et al. Immunization rates and vaccine beliefs among patients with inflammatory bowel disease: an opportunity for improvement. Inflamm Bowel Dis 2014;20:246-50.

4. Auriel E, Gadoth A, Regev K, et al. Seasonal and H1N1v influenza vaccines in MS: safety and compliance. 7 Neurol Sci 2012;314:102-3.

5. Subesinghe S, Rutherford AI, Ibrahim F, et al. A large two-centre study in to rates of influenza and pneumococcal vaccination and infection burden in rheumatoid arthritis in the UK. BMC Musculoskelet Disord 2016;17:322.

6. Martens PJ, Chochinov HM, Prior HJ, et al.; Need To Know Team. Are cervical cancer screening rates different for women with schizophrenia? A Manitoba population-based study. Schizophr Res 2009;113:101-6.

7. Lord O, Malone D, Mitchell AJ. Receipt of preventive medical care and medical screening for patients with mental illness: a comparative analysis. Gen Hosp Psychiatry 2010;32:519-43.

8. Marrie RA, Walld R, Bolton JM, et al.; CIHR Team in Defining the Burden and Managing the Effects of Psychiatric Comorbidity in Chronic Immunoinflammatory Disease. Increased incidence of psychiatric disorders in immune-mediated inflammatory disease. 7 Psychosom Res 2017;101:17-23.

9. Pham HV, Hasan I, Udaltsova N, et al. Rates and predictors of vaccinations among inflammatory bowel disease patients receiving anti-tumor necrosis factor agents. Dig Dis Sci 2018;63:209-17.

10. Bernstein CN, Wajda A, Svenson LW, et al. The epidemiology of inflammatory bowel disease in Canada: a population-based study. Am 7 Gastroenterol 2006;101:1559-68.

11. Artbritis in Canada: an ongoing challenge. Ottawa: Health Canada; 2003. Cat no H39-4/14-2003E.

12. Evans C, Beland SG, Kulaga S, et al. Incidence and prevalence of multiple sclerosis in the Americas: a systematic review. Neuroepidemiology 2013;40:195-210.

13. Roos LL, Mustard CA, Nicol JP. Registries and administrative data: organization and accuracy. Med Care 1993;31:201-12. 
14. Roos LL, Sharp SM, Cohen MM. Comparing clinical information with claims data: some similarities and differences. 7 Clin Epidemiol 1991:44:881-8.

15. Roos LL, Walld R, Wajda A, et al. Record linkage strategies, outpatient procedures, and administrative data. Med Care 1996;34:570-82.

16. ICD-9-CM: international classification of diseases, 9th revision, clinical modification. 5th ed. Medicode; 1996.

17. ICD-10-CA: international statistical classification of diseases and related bealth problems, tenth revision, Canada. Ottawa: Canadian Institute for Health Information; 2015.

18. Anatomic therapeutic chemical classification system - structure and principles. WHO Collaborating Centre for Drug Statistics Methodology; updated 2018 Feb. 15. Available: www.whocc.no/atc/structure_and_principles/ (accessed 2012 Aug. 9).

19. Bernstein CN, Blanchard JF, Rawsthorne P, et al. Epidemiology of Crohn's disease and ulcerative colitis in a central Canadian province: a populationbased study. Am 7 Epidemiol 1999;149:916-24.

20. Al-Sakran LH, Marrie RA, Blackburn DF, et al. Establishing the incidence and prevalence of multiple sclerosis in Saskatchewan. Can 7 Neurol Sci 2018;45:295-303.

21. Hitchon CA, Khan S, Elias B, et al. Prevalence and incidence of rheumatoid arthritis in Canadian First Nations and non-First Nations people: a population-based study. 7 Clin Rheumatol 2020;26:169-75.

22. Marrie RA, Fisk JD, Yu BN, et al.; CIHR Team in the Epidemiology and Impact of Comorbidity on Multiple Sclerosis. Mental comorbidity and multiple sclerosis: validating administrative data to support population-based surveillance. BMC Neurol 2013;13:16.

23. Starfield B, Weiner J, Mumford L, et al. Ambulatory care groups: a categorization of diagnoses for research and management. Health Serv Res 1991; 26:53-74.

24. Weiner JP, Starfield BH, Steinwachs DM, et al. Development and application of a population-oriented measure of ambulatory care case-mix. Med Care 1991;29:452-72.

25. Reid RJ, Roos NP, MacWilliam L, et al. Assessing population health care need using a claims-based ACG morbidity measure: a validation analysis in the province of Manitoba. Health Serv Res 2002;37:1345-64.

26. Sjölander A, Greenland S. Ignoring the matching variables in cohort studies - When is it valid and why? Stat Med 2013;32:4696-708.

27. Aday LA, Andersen R. A framework for the study of access to medical care. Health Serv Res 1974;9:208-20.

28. Andersen RM. National health surveys and the behavioral model of health services use. Med Care 2008;46:647-53.

29. Andersen R, Aday LA, Chen MS. Health status and health care utilization. Health Aff (Millwood) 1986;5:154-72.

30. Chateau D, Metge C, Prior H, et al. Learning from the census: the Socioeconomic Factor Index (SEFI) and health outcomes in Manitoba. Can 7 Public Health 2012;103:S23-7.

31. Rothman KJ, Greenland S. Matching. In: Rothman KJ, Greenland S, editors. Modern epidemiology. 2nd ed. Philadelphia: Lippincott Williams \& Wilkins; 1998:147-61.

32. Farraye FA, Melmed GY, Lichtenstein GR, et al. ACG clinical guideline: preventive care in inflammatory bowel disease. Am 7 Gastroenterol 2017;112:241-58.

33. Farez MF, Correale J, Armstrong MJ, et al. Practice guideline update summary: vaccine-preventable infections and immunization in multiple sclerosis: report of the guideline development, dissemination, and implementation subcommittee of the American Academy of Neurology. Neurology 2019; 93:584-94.

34. Furer V, Rondaan C, Heijstek MW, et al. 2019 update of EULAR recommendations for vaccination in adult patients with autoimmune inflammatory rheumatic diseases. Ann Rheum Dis 2020;79:39-52.

35. Roy M, Sherrard L, Dubé È, et al. Determinants of non-vaccination against seasonal influenza. Health Rep 2018;29:12-22.

36. Boltin D, Gingold-Belfer R, Kimchi NA, et al. Utilization of influenza immunization in adults with Crohn's disease-a longitudinal, populationbased study. Inflamm Bowel Dis 2014;20:240-5.

37. Boltin D, Gingold-Belfer R, Kimchi NA, et al. Uptake of influenza vaccine in ulcerative colitis. Vaccine 2014;32:5484-9.

38. Qendro T, de la Torre ML, Panopalis P, et al. Suboptimal immunization coverage among Canadian rheumatology patients in routine clinical care. 7 Rheumatol 2020;47:770-8.

39. Costello R, Winthrop KL, Pye SR, et al. Influenza and pneumococcal vaccination uptake in patients with rheumatoid arthritis treated with immunosuppressive therapy in the UK: a retrospective cohort study using data from the clinical practice research datalink. PLoS One 2016;11:e0153848.

40. Krasselt M, Ivanov JP, Baerwald C, et al. Low vaccination rates among patients with rheumatoid arthritis in a German outpatient clinic. Rheumatol Int 2017;37:229-37.

41. Ghaderi S, Berg-Hansen P, Bakken IJ, et al. Hospitalization following influenza infection and pandemic vaccination in multiple sclerosis patients: a nationwide population-based registry study from Norway. Eur F Epidemiol 2020;35:355-62.

42. Szilagyi PG, Shone LP, Barth R, et al. Physician practices and attitudes regarding adult immunizations. Prev Med 2005;40:152-61.

43. Nigro G, Angelini G, Grosso SB, et al. Psychiatric predictors of noncompliance in inflammatory bowel disease: psychiatry and compliance. 7 Clin Gastroenterol 2001;32:66-8.
44. Mohr DC, Goodkin DE, Likosky W, et al. Treatment of depression improves adherence to interferon beta-1b therapy for multiple sclerosis. Arch Neurol 1997;54:531-3

45. Tarrants M, Oleen-Burkey M, Castelli-Haley J, et al. The impact of comorbid depression on adherence to therapy for multiple sclerosis. Mult Scler Int 2011;2011:271321.

46. DiMatteo MR, Lepper HS, Croghan TW. Depression is a risk factor for noncompliance with medical treatment: meta-analysis of the effects of anxiety and depression on patient adherence. Arch Intern Med 2000; 160:2101-7.

47. Farmanara N, Sherrard L, Dubé È, et al. Determinants of non-vaccination against seasonal influenza in Canadian adults: findings from the 2015-2016 Influenza Immunization Coverage Survey. Can 7 Public Health 2018;109:369-78.

48. Malhi G, Rumman A, Thanabalan R, et al. Vaccination in inflammatory bowel disease patients: attitudes, knowledge, and uptake. 7 Crobns Colitis 2015;9:439-44.

49. Hammami MB, Pandit P, Salamo RT, et al. Health maintenance and vaccination of patients with inflammatory bowel disease: practice and perception of responsibility of gastroenterologists vs primary care providers. Ochsner $f$ 2019;19:210-9.

50. Ledwich LJ, Harrington TM, Ayoub WT, et al. Improved influenza and pneumococcal vaccination in rheumatology patients taking immunosuppressants using an electronic health record best practice alert. Arthritis Rheum 2009;61:1505-10.

51. Parker S, Chambers White L, Spangler C, et al. A quality improvement project significantly increased the vaccination rate for immunosuppressed patients with IBD. Inflamm Bowel Dis 2013;19:1809-14.

52. Shah SC, Khalili H, Gower-Rousseau C, et al. Sex-based differences in incidence of inflammatory bowel diseases - pooled analysis of population-based studies from western countries. Gastroenterology 2018;155:1079-89.e3.

53. Dobson R, Giovannoni G. Multiple sclerosis - a review. Eur 7 Neurol 2019;26:27-40.

54. Minichiello E, Semerano L, Boissier MC. Time trends in the incidence, prevalence, and severity of rheumatoid arthritis: a systematic literature review. Foint Bone Spine 2016;83:625-30.

55. Gionet L. Flu vaccination rates in Canada. Ottawa: Statistics Canada; 2015. Cat no 82-624-X.

Competing interests: For work outside the study reported here, Ruth Ann Marrie has received research funding from the Canadian Institutes of Health Research (CIHR), Research Manitoba, the Multiple Sclerosis Society of Canada, the Multiple Sclerosis Scientific Research Foundation, Crohn's and Colitis Canada, the National Multiple Sclerosis Society and the Consortium of Multiple Sclerosis Centers; she has also participated in research funded by Roche and Biogen Idec (all funds to co-investigators). Jitender Sareen has received consulting fees from UpToDate and previously held stock in Johnson \& Johnson. For work outside the study reported here, Scott Patten has received research funding from CIHR, the Hotchkiss Brain Institute and the Multiple Sclerosis Society of Canada (which includes contributions from Roche, Biogen and the Government of Alberta); he also holds the Cuthbertson and Fischer Chair in Pediatric Mental Health at the University of Calgary. Alexander Singer has received financial and in-kind support from an IBM/CIMVHR Advanced Analytics Grant and Calian Inc. For work outside the study reported here, Lisa Lix has received research funds from CIHR and the Arthritis Society. Carol Hitchon has received research funds for unrelated studies from UCB Canada and Pfizer. James Marriott has received grant funding from Roche (as site principal investigator for a clinical trial). Renée El-Gabalawy has received research funds for unrelated studies from University of Manitoba Start-Up Funds, the CIHR Chronic Pain Network, Health Sciences Centre foundation grant, Department of Anesthesia operating grant and the Tri-Agency New Frontiers in Research Fund. For activities unrelated to the current study, John Fisk has received research funds from CIHR, the Multiple Sclerosis Society of Canada, the Nova Scotia Health Authority Research Fund and the Dalhousie Medical Research Fund, as well as royalty fees from MAPI Research Trust. For activities unrelated to the current study, Charles Bernstein has received consultancy fees from Roche Canada, Mylan Pharmaceuticals and Takeda Canada; contract research funding from Pfizer, Janssen Canada and Roche; unrestricted educational or research grants from Abbvie Canada, Janssen Canada, Pfizer Canada, Takeda Canada, Sandoz and Medtronic Canada; speakers' fees from Abbvie Canada, Janssen Canada, Pfizer Canada, Takeda Canada and Medtronic Canada; and has served on advisory boards for Abbvie Canada, Janssen Canada, Pfizer Canada, Takeda Canada, Sandoz, Amgen Canada, Bristol Myers Squibb Canada and Roche Canada. No other competing interests were declared. 
Affiliations: Department of Internal Medicine (Marrie, Hitchon, Marriott, Bernstein), Department of Community Health Sciences (Marrie, Lix, Katz), Manitoba Centre for Health Policy (Walld, Katz), Department of Psychiatry (Bolton, Sareen), Department of Family Medicine (Singer, Katz), Department of Clinical Health Psychology (El-Gabalawy) and Department of Anesthesiology and Perioperative and Pain Medicine (El-Gabalawy), Max Rady College of Medicine, Rady Faculty of Health Sciences, University of Manitoba, Winnipeg, Man.; Department of Community Health Sciences (Patten), Cumming School of Medicine, University of Calgary, Calgary, Alta.; Departments of Psychiatry (Fisk), of Psychology and Neuroscience (Fisk) and of Medicine (Fisk), Dalhousie University, Halifax, NS

Contributors: Ruth Ann Marrie, James Bolton, Jitender Sareen, Scott Patten, Lisa Lix, Carol Hitchon, James Marriott, Alan Katz, John Fisk and Charles Bernstein obtained study funding and designed the study. Randy Walld analyzed the data, and all of the authors interpreted the data. Ruth Ann Marrie drafted the manuscript, and Randy Walld, James Bolton, Jitender Sareen, Scott Patten, Alexander Singer, Lisa Lix, Carol Hitchon, James Marriott, Renée El-Gabalawy, Alan Katz, John Fisk and Charles Bernstein revised the manuscript for important intellectual content. All of the authors approved the final version for publication and agreed to be accountable for the work.

Members of the CIHR Team in Defining the Burden and Managing the Effects of Psychiatric Comorbidity in Chronic Immunoinflammatory Disease: Ruth Ann Marrie, James M. Bolton, Jitender Sareen, John R. Walker (deceased), Scott B. Patten, Alexander Singer, Lisa M. Lix, Carol A. Hitchon, Renée El-Gabalawy, Alan Katz, John D. Fisk, Charles N. Bernstein, Lesley Graff, Lindsay Berrigan, Ryan Zarychanski, Christine Peschken, James J. Marriott.

Funding: This study was funded by the Canadian Institutes of Health Research (CIHR; THC-135234), Crohn's and Colitis Canada and the Waugh Family Chair in Multiple Sclerosis (to Ruth Ann Marrie). James Bolton has received research funding from the Brain \& Behavior
Research Foundation and the Multiple Sclerosis Society of Canada. Charles Bernstein is supported in part by the Bingham Chair in Gastroenterology. Jitender Sareen is supported by CIHR grant no. 333252. Lisa Lix is supported by a Canada Research Chair. Renée El-Gabalawy is supported by University of Manitoba Start-Up Funding. Alan Katz held a contract from the Government of Manitoba for maintenance of the data repository. The sponsors had no role in the design and conduct of the study; the collection, management, analysis or interpretation of the data; or the preparation, review or approval of the manuscript.

Content licence: This is an Open Access article distributed in accordance with the terms of the Creative Commons Attribution (CC BY-NCND 4.0) licence, which permits use, distribution and reproduction in any medium, provided that the original publication is properly cited, the use is noncommercial (i.e., research or educational use), and no modifications or adaptations are made. See: https://creativecommons.org/licenses/ by-nc-nd/4.0/

Data sharing: The authors of this study are not the data custodians and are therefore not authorized to make the study data available to others. With appropriate approvals, the data can be accessed through the Manitoba Centre for Health Policy.

Acknowledgements: The Aggregated Diagnosis Groups (ADGs) codes for risk adjustment in regression models were created using The Johns Hopkins Adjusted Clinical Group (ACG) Case-Mix System version 9. The authors acknowledge the Manitoba Centre for Health Policy for use of the Population Health Research Data Repository under project \#2014030 (HIPC \#2014/2015-19A). The results and conclusions presented are those of the authors; no official endorsement by the Manitoba Centre for Health Policy, Manitoba Health or other data providers is intended or should be inferred.

Supplemental information: For reviewer comments and the original submission of this manuscript, please see www.cmajopen.ca/content/9/2/ E510/suppl/DC1. 